\title{
Arztpraxis als Aktiengesellschaft: Wie hoch ist der angemessene Lohn?
}

\author{
Martin Müllera, Paul Müller-Wittwer ${ }^{\mathrm{b}}$ \\ ${ }^{a}$ Bücherexperte VSB, dipl. Steuerexperte; ${ }^{b}$ Rechtsanwalt, dipl. Steuerexperte
}

Ärztinnen und Ärzte, die sich für eine Arztpraxis in der Rechtsform einer Aktiengesellschaft (oder $\mathrm{GmbH}$ ) entscheiden, fragen sich: Wie sollen wir unseren Lohn festlegen?

Die AHV-Ausgleichskasse medisuisse hat im Gerichtsfall, der vorliegend besprochen wird, eine Praxisverschärfung einleiten wollen. Dividenden, die mehr als $10 \%$ des Steuerwertes der Aktien betragen, sollten neu automatisch als massgebender Lohn gelten.

Das Bundesgericht hat am 24. Januar 2019 (9C_4/2018) diese Praxisverschärfung abgelehnt. Es hat jedoch gleichzeitig akzeptiert, dass ein Teil der Dividende der AHV-Beitragspflicht unterstellt worden ist.

\section{Résumé}

Les médecins qui optent pour un cabinet médical sous la forme juridique de société anonyme ou société à responsabilité limitée se posent la question suivante: comment devrions-nous déterminer notre salaire?

Le niveau de salaire choisi revêt une grande importance, par exemple pour la couverture d'assurance, les questions liées au droit de la famille, les questions entre actionnaires, le montant des frais acceptés, l'impôt sur la fortune, l'impôt sur le bénéfice et le revenu ainsi que le montant des cotisations AVS.

Lorsque les sociétés de conseil comparent la société anonyme à d'autres formes juridiques, elles se fondent sur un certain rapport entre la rémunération et les dividendes. Dans ces comparaisons, aucune cotisation AVS n'est calculée sur le dividende.

La caisse de compensation AVS medisuisse a voulu introduire une pratique plus stricte dans le cas discuté ici. Les dividendes supérieurs à $10 \%$ de la valeur imposable des actions devraient automatiquement être considérés comme salaire déterminant.

Le 24 janvier 2019 (9C_4/2018), le Tribunal fédéral a rejeté ce durcissement de la pratique, car il est manifestement contraire à la volonté législative. Dans le même temps, il a toutefois accepté qu'une partie du dividende soit soumise à I'obligation de contribution AVS.

Pour un médecin spécialiste en médecine interne générale, un salaire de 169000 francs suisses (converti à 100\%) a été jugé trop bas. Un salaire de 208000 francs suisses était soumis à l'obligation de cotisation AVS.

En discutant de ce jugement important, nous voudrions montrer aux médecins comment fixer des salaires pouvant être acceptés par la medisuisse et par les tribunaux.
Mit der Besprechung dieses wichtigen Urteils möchten wir den Ärztinnen und Ärzten aufzeigen, wie ein Lohn festgelegt wird, der schlussendlich von der medisuisse und den Gerichten akzeptiert werden kann.

Zum besseren Verständnis wurden Angaben zum Sachverhalt vereinfacht und dort, wo in der Urteilsbegründung Angaben fehlen, Annahmen getroffen.

\section{Sachverhalt}

Zwei Ärzte (Dr. B und Dr. C) haben ab 2008 eine Gemeinschaftspraxis in der Rechtsform einer Aktiengesellschaft (AG) betrieben. Dr. B war Facharzt für Allgemeine Innere Medizin. Dr. $\mathrm{C}$ hatte einen anderen Facharzttitel. Dies war für das Urteil jedoch unwesentlich.

Beide Ärzte waren zuvor selbständig erwerbend und erzielten daraus ein Einkommen zwischen 310000 CHF und $520000 \mathrm{CHF}$.

In der AG legten sie den Lohn im Jahr 2009 auf je 144000 CHF fest. Dies bei einem Pensum von je $85 \%$ (d.h. bei 100\% rund $169000 \mathrm{CHF}$ ).

Die AG erzielte im Geschäftsjahr 2009 einen Reingewinn von 500000 CHF. Sie schüttete an Dr. B und Dr. C für dieses Geschäftsjahr je eine Dividende von $250000 \mathrm{CHF}$ aus.

In der AG arbeiteten in der Folge noch andere Fachärzte, insbesondere Dr. D und Dr. E:

- Dr. D war 2014 noch ein Praxisassistent im vierten Ausbildungsjahr. Ab dem 1. Mai 2016 war er als Facharzt für Allgemeine Innere Medizin tätig und übernahm die Praxisvertretung im Vollzeitpensum. Er erzielte umgerechnet einen Jahresbruttolohn von 150000 CHF.

- Dr. E arbeitete als Fachärztin für Innere Medizin mit einem Pensum von 50\%. Der auf ein Vollzeitpensum umgerechnete Lohn betrug 168000 CHF im Jahr 2015 und 200000 CHF im Jahr 2016.

\section{Die medisuisse will Praxisverschärfung}

Die medisuisse erachtete den von der AG für Dr. B und Dr. C für das Jahr 2009 festgelegten Lohn als zu tief. Sie 
verglich diesen Lohn zuerst mit demjenigen, den die Angestellten Dr. D und Dr. E in den Jahren 2015 und 2016 verdienten. Aufgrund dieses Vergleichs hätte ihres Erachtens der Lohn von Dr. B und Dr. C bei einem Vollzeitpensum mindestens 208000 CHF betragen müssen. Auf das Teilzeitpensum (85\%) von Dr. A bzw. Dr. B umgerechnet, musste der Lohn damit je 177000 CHF betragen, d.h. 33000 CHF mehr als die effektiven $144000 \mathrm{CHF}$.

Die medisuisse verglich anschliessend die Dividende mit dem Steuerwert der Aktien per 31.12.2009. Denn nach der Praxis des Bundesamtes für Sozialversicherung sind Dividenden von mehr als 10\% des Steuerwertes der Aktien vermutungsweise überhöht.

Der Steuerwert der Aktien betrug insgesamt ungefähr 3800000 CHF. Daraus wurde abgeleitet, dass eine Dividende von mehr als 190000 CHF für Dr. B bzw. Dr. C vermutungsweise überhöht sei. Damit war nach Auffassung der medisuisse die Dividende je um 60000 CHF überhöht.

Die medisuisse machte vor Bundesgericht geltend, dass der massgebende Lohn von Dr. B und Dr. C nicht nur um 33000 CHF, sondern um 60000 CHF zu erhöhen sei. Auf das Kriterium des Missverhältnisses zwischen Arbeitsleistung und Lohn sei zu verzichten. Dieses sei nicht praktikabel und letztlich willkürlich.

\section{Nach welchen Grundsätzen wird ein Lohn überprüft?}

Das Bundesgericht legte zuerst die anwendbaren Grundsätze dar. Vom Einkommen aus unselbständiger Erwerbstätigkeit wird ein AHV-Beitrag erhoben. Dieses Einkommen wird auch «massgebender Lohn» genannt. Dividenden und anderer Kapitalertrag auf Aktien, die sich im Privatvermögen befinden, unterliegen keinem AHV-Beitrag. Ob eine Vergütung einer AG als Kapitalertrag zu qualifizieren ist, beurteilt sich danach, ob sie ihren (wahren) Grund im Arbeitsverhältnis hat oder nicht.

Von der durch die AG gewählten Aufteilung zwischen Lohn und Dividende dürfen die Behörden nur abweichen, wenn kumulativ zwei Voraussetzungen erfüllt sind:

- ein offensichtliches Missverhältnis zwischen Arbeitsleistung und Lohn

- ein offensichtliches Missverhältnis zwischen eingesetztem Vermögen und Dividende

Die Gesellschaft hat bei der Bestimmung von Lohn und Dividende einen erheblichen Ermessensspielraum. Den Behörden steht nicht zu, die Angemessenheit des
Lohnes bzw. der Dividende frei zu überprüfen. Dies hat das Bundesgericht in einem anderen Urteil erwähnt (vgl. Urteil des Bundesgerichts vom 29. Mai 2017, 9C_89/2017, E. 4.2.1).

Praxisgemäss ist es zwar Sache der Ausgleichskassen, selbständig zu beurteilen, ob eine Vergütung massgebenden Lohn darstellt. Es entspricht jedoch der AHV-

Den Behörden steht nicht zu, die Angemessenheit des Lohnes bzw. der Dividende frei zu überprüfen.

rechtlichen Ordnung, dass sie sich in der Regel an die bundessteuerrechtliche Betrachtungsweise halten. Soweit vertretbar, soll eine unterschiedliche Einschätzung der Steuerbehörde und der AHV-Verwaltung vermieden werden, damit die Einheit der Rechtsordnung gewahrt bleibt.

\section{Arbeitsleistung und Lohn}

Das Bundesgericht überprüfte nun, ob ein offensichtliches Missverhältnis zwischen Arbeitsleistung und Lohn besteht.

Es hat in diesem Zusammenhang die Auffassung des kantonalen Gerichts wiedergegeben, dass nicht ohne weiteres auf das Einkommen aus selbständiger Erwerbstätigkeit abzustellen sei, das Dr. B und Dr. C in den Jahren 2003 bis 2007 erzielt haben.

Das unternehmerische Risiko als selbständig erwerbende Person sei ein grundlegend anderes als dasjenige eines Arbeitnehmers. Die Entschädigung für dessen Tragung komme nicht dem Arbeitnehmer, sondern dem Inhaber der Beteiligungsrechte per Dividende zu.

Das Bundesgericht erachtete es jedoch als nicht willkürlich, dass das kantonale Gericht:

- den Lohn von Dr. B und Dr. C mit demjenigen von Dr. D und Dr. E verglich (= innerbetrieblicher Vergleich);

- den Lohn von Angestellten, die nicht annähernd mit Dr. B und Dr. C vergleichbar sind, ausgeklammert hat (z.B. Assistenzärzte und Medizinische Praxisassistentinnen);

- den Lohn in den Jahren 2015 und 2016 (Dr. D bzw. Dr. E) mit dem Lohn im Jahr 2009 (Dr. B bzw. Dr. C) verglich, denn es wurde von der AG nicht geltend gemacht, dass sich die Sachlage massgeblich geändert hat;

- im Ergebnis ein offensichtliches Missverhältnis zwischen Arbeitsleistung und Lohn von je 33000 CHF angenommen hat. 
In der Volksabstimmung vom 19. Mai 2019 wurde auf Bundesebene eine Steuerreform angenommen. Danach wird eine Dividende neu zu 70\% als steuerbares Einkommen erfasst. Diese Änderung tritt voraussichtlich am 1. Januar 2020 in Kraft. Auch in zahlreichen Kantonen sind in diesem Zusammenhang Steuerreformen eingeleitet, bereits beschlossen oder (vorerst) abgelehnt worden. Diese Steuerreformen können dazu führen, das sich die Attraktivität einer AG bzw. des Dividendenbezugs ändert. An den praktischen Empfehlungen, wie der Lohn in einer AG festgelegt werden sollte, ändert sich allein deswegen nichts. Die gesamte Abgabebelastung ist jedoch ein Kriterium das in Abwägung mit den anderen Kriterien angemessen mitberücksichtigt werden sollte.

Korrespondenz: mmberatung ag Paul Müller-Wittwe Seilerstrasse 4 CH-3011 Bern

Tel. 0313858000 pm[at]mmberatung.ch www.mmberatung.ch

\section{Vermögen und Dividende}

Anschliessend überprüfte das Bundesgericht, ob auch ein offensichtliches Missverhältnis zwischen eingesetztem Vermögen und Dividende besteht. Dieses Missverhältnis beurteilt sich jeweils in Relation zum effektiven wirtschaftlichen Wert der Aktien.

Die Praxis des Bundesamtes für Sozialversicherung, nach der ein offensichtliches Missverhältnis vermutet wird, wenn die Dividende mehr als 10\% des Steuerwertes der Aktien beträgt, wurde nicht beanstandet. Vorliegend hatte die AG nichts gegen diese Vermutung vorgebracht. Für das Bundesgericht war darum die Feststellung des kantonalen Gerichts nicht willkürlich, dass je 60000 CHF der Dividende überhöht gewesen seien.

\section{Entscheid: Keine Verschärfung der Praxis}

Gemäss der Unternehmenssteuerreform II werden seit dem 1. Januar 2009 Dividenden aufgrund von Aktien (im Privatvermögen) auf Bundesebene nur noch zu $60 \%$ als steuerbares Einkommen erfasst. Damit wurde die wirtschaftliche Doppelbelastung des Gewinns der Kapitalgesellschaften gemildert. Auch die Kantone haben in unterschiedlichem Umfang Milderungen eingeführt. Der Gesetzgeber hat sich im Wissen um die Auswirkungen auf die AHV für die Teilbesteuerung der Dividenden entschieden ${ }^{1}$.

Das Bundesgericht weist darauf hin, dass der Antrag, Dividenden über 10\% des Steuerwertes der Aktien der AHV-Beitragspflicht zu unterwerfen, im Parlament ausdrücklich abgelehnt worden ist.

Wenn nun die AHV-Ausgleichskasse das Beitragssubstrat auf dem Weg der Rechtsprechungsänderung ausweiten wolle, entspreche dies offensichtlich nicht dem gesetzgeberischen Willen.

Im Ergebnis entschied das Bundesgericht wie die Vorinstanz. Es schützte, dass bei Dr. B und Dr. C im Jahr 2009 der massgebende Lohn um je 33000 CHF erhöht worden ist. Es verwarf jedoch den Versuch, eine Aufrechnung von je 60000 CHF vorzunehmen.

\section{Praktische Empfehlungen}

Eine AG hat damit weiterhin einen erheblichen Ermessensspielraum. Der vorliegende Fall zeigt aber, dass bei der Festlegung des Lohnes Vorsicht angezeigt ist. Es ist ratsam, den Lohn umsichtig festzulegen.

Wie soll nun aber eine AG bestimmen, wie hoch der Lohn einer beteiligten Ärztin oder eines beteiligten Arztes sein soll? Wir empfehlen, dass vier Überlegungen angestellt werden:

- In welcher Bandbreite wäre der Lohn, den man für eine vergleichbare Tätigkeit in einer anderen $\mathrm{Ge}$ sellschaft fordern könnte und fordern würde, an der man nicht beteiligt ist?

- Ist der Lohn im innerbetrieblichen Vergleich nachvollziehbar?

- Befindet sich der Lohn innerhalb einer vernünftigen Bandbreite der Löhne, die Ärztinnen und Ärzte in anderen Arztpraxen und Spitälern verdienen? Dabei sollte auf eine genügende Vergleichbarkeit geachtet werden.

- Ist die gewählte Lohnhöhe auch bei einer Gesamtbetrachtung umsichtig gewählt worden?

Die Lohnhöhe ist z.B. regelmässig von Bedeutung für den Versicherungsschutz, familienrechtliche Themen, Fragen unter Aktionären, den Vermögenssteuerwert, ein akzeptables Niveau von Spesen, für die Höhe von AHV-Beiträgen sowie Gewinn- und Einkommenssteuern. Je nach Situation kann es empfehlenswert sein, wenn man versucht, sich vorgängig mit der AHVAusgleichskasse abzustimmen.

Wenn die revidierende Person in der Arbeitgeberkontrolle zur Auffassung neigt, dass ein Lohn im geforderten Umfang unangemessen tief ist, so erachten wir die rückwirkende Erhöhung des massgebenden Lohnes im Normalfall nicht als das sachgerechteste Vorgehen. Vorzugswürdig wäre, wenn im gemeinsamen Gespräch für die Zukunft ein Lohn festgelegt wird, der für beide Seiten als angemessen gelten kann. Ein solches Vorgehen wahrt die Einheit der Rechtsordnung, reduziert Gerichtsverfahren und fördert die gegenseitige Wertschätzung. 